\title{
Pessoas com deficiência: entre necessidades e atenção à saúde ${ }^{1}$
}

\author{
Fernanda dos Reis Souza ${ }^{\mathrm{a}}$, Adriana Miranda Pimentel ${ }^{\mathrm{b}}$
}

\begin{abstract}
aTerapeuta Ocupacional, Mestranda em Saúde Pública, Universidade Federal do Ceará - UFC, Fortaleza, CE, Brasil 'Professora Doutora, Saúde Coletiva, Instituto de Humanidades, Artes e Ciências Professor Milton Santos, Universidade Federal da Bahia - UFBA, Salvador, BA, Brasil
\end{abstract}

\begin{abstract}
Resumo: Este estudo traça o perfil das pessoas com deficiência e suas necessidades de saúde em um bairro da cidade de Salvador, Bahia. Neste, buscou-se conhecer a situação de vida e saúde das pessoas com deficiência a partir do contexto onde vivem. A investigação foi realizada em duas áreas de cobertura do Programa de Agentes Comunitários de Saúde nas quais foram aplicados questionários abertos com pessoas com deficiência física e sensorial. Foram selecionadas variáveis sócio-demográficas, bem como acesso a serviços e projetos de vida futuros. Os resultados revelam uma população em que predominam adultos e idosos, negros, do sexo feminino, com renda média entre meio e três salários mínimos, baixa escolaridade e com dificuldades em relação ao acesso a serviços de saúde no bairro e na cidade. As pessoas referem necessidade de atendimento especializado de saúde no bairro, melhorias nas condições de vida e expressam o desejo no que tange ao retorno de atividades que realizavam antes da deficiência. Tais dados evidenciam uma população caracterizada por um complexo multifacetado de exclusão e privação coletiva e apontam para a necessidade de compreensão dos processos sociais na melhoria da assistência oferecidas às populações. Para isso, aponta-se a reabilitação na Atenção Primária à Saúde como estratégia de relevância para mudanças na atenção à saúde das pessoas com deficiência.

Palavras-chave: Pessoas com Deficiência, Necessidades e Demandas de Serviços de Saúde, Atenção Primária à Saúde.
\end{abstract}

\section{People with disabilities: between needs and health care}

\begin{abstract}
This study traces the profile of people with disabilities, as well as their health needs, in a neighborhood of Salvador, State of Bahia. In this, the life and health conditions of people with disabilities in the context they live were studied. The research was carried out in two coverage areas of the Community Health Workers Program ("Programa de Agentes Comunitários de Saúde"), where open-ended questionnaires were conducted with people with physical and sensory disabilities. Socio-demographic variables on disability and access to services and projects for future life were selected. Results showed that the population is comprised predominantly of adults and elderly, African descent, female, with average income between half and three minimum wages, poorly educated, presenting difficulties in relation to access to health services in the neighborhood and the city. People with disabilities refer to the need of specialized health care in the neighborhood, improvements in life conditions, and expressed the desire to resume the activities they used to perform before the disability. These data reveal a population characterized by a multifaceted complex of collective exclusion and deprivation and point to the need for understanding social processes when improving the assistance offered to the population. To this end, it is necessary to rehabilitate the Primary Health Care as a strategy of relevance to changes in health care for people with disabilities.
\end{abstract}

Keywords: People with Disabilities, Health Services Needs and Demand, Primary Health Care. 


\section{Introdução}

As pessoas com deficiência foram historicamente segregadas e excluídas dos processos sociais. O olhar sobre essa populaçáo variava de acordo com a cultura, com o período histórico e com os valores de cada sociedade. Na realidade, cada

[...] sociedade estabelece meios de categorizar as pessoas e o total de atributos considerados como comuns e naturais para os membros de cada uma dessas categorias. (GOFFMAN, 1963, p. 12).

A partir da categorização, surgem os atributos estranhos, aqueles diferentes dos demais, que o autor denomina de estigma. O indivíduo estigmatizado é, portanto, aquele que possui diferenças em relação à dita normalidade de uma sociedade, como por exemplo, as pessoas com deficiência. Apenas recentemente a problemática das pessoas com deficiência saiu da esfera privada para a vida pública, tendo suas demandas reconhecidas como uma questáo de direitos humanos (DINIZ; BARBOSA; SANTOS, 2009), contudo ainda hoje, estâo submetidas a processos de privação coletiva, ou exclusão social, o que nos faz compreender que a problemática da deficiência náo está dissociada do contexto sociocultural.

Este trabalho tomou como ponto de partida os conceitos da Classificação Internacional de Funcionalidade, Incapacidade e Saúde (CIF), por entender que a funcionalidade dos indivíduos é determinada pelo contexto em que as pessoas vivem. Segundo essa classificação, a

[...] funcionalidade e a incapacidade de uma pessoa são concebidas como uma interacção dinâmica entre os estados de saúde (doenças, perturbações, lesôes, traumas, etc.) e os factores contextuais. (ORGANIZAÇÃO..., 2003, p. 10).

Assim, deficiências são problemas nas funçôes ou nas estruturas do corpo, tais como, um desvio importante ou uma perda, que pode envolver uma situação de limitação nas atividades, que consiste na execução de uma tarefa ou ação por um indivíduo, e na participação, que é o envolvimento de um indivíduo numa situação da vida real (ORGANIZAÇÃO..., 2003).

As pessoas com deficiência correspondem, conforme o Censo 2000, a 14,5\% da população do país. Em um levantamento da Organização Pan-Americana de Saúde (OPAS), identificou-se que em todos os países (tanto os desenvolvidos quanto os em desenvolvimento) o predomínio de incapacidades aumenta nas áreas mais marginalizadas (MAIOR, 1997). Santos, Silva e Lessa (1989), em um estudo com dois grupos sociais diferentes em Salvador, comprovam que a prevalência de incapacidade física era de 6,2\% para os favelados e $1,8 \%$ entre os não-favelados. $\mathrm{O}$ fato de as pessoas com deficiência serem as mais pobres entre os pobres, em todas as partes do mundo, leva à reflexão sobre a dupla exclusão social a qual estão submetidas essas pessoas

$\mathrm{Na}$ saúde, com o processo de construção do Sistema Único de Saúde (SUS) a partir de 1988, importantes avanços aconteceram no que concerne à garantia de direitos, como o reconhecimento do dever do Estado de disponibilizar serviços de saúde e reabilitação para as pessoas com deficiência. Contudo, sabe-se que a criação do SUS se deu sob conflitos e contradiçôes, de forma que, ainda hoje, o sistema de saúde brasileiro vive um cenário de disputas entre diferentes modelos assistenciais, com tendência a reproduzir a lógica hegemônica, sob a égide da assistência privada, na tentativa de construção de um modelo alternativo para a superação desta lógica (TEIXEIRA; PAIM; VILASBOAS, 1998).

A análise da assistência à saúde das pessoas com deficiência aponta um perfil de fragilidade, desarticulação e descontinuidade de açóes nas esferas pública e privada (BRASIL, 2002) e com baixo índice de cobertura da assistência. Segundo a Organização Mundial de Saúde (OMS), apenas $2 \%$ dos portadores de deficiência teriam acesso à reabilitaçáo; nos países em desenvolvimento esse número é de 1 a 2 por 10.000 habitantes. Na cidade de Salvador, verifica-se que a atenção a esse grupo se dá, predominantemente, no último nível de atenção do sistema, fato que se revela em desacordo com a Política Nacional da Pessoa com deficiência, que preconiza a atenção nos diferentes níveis de complexidade do sistema. A atenção no último nível (representada pelos centros de reabilitação) está fundada numa visão mecanicista do corpo, possuindo como objetivo uma intervenção em que o desempenho funcional deve ser corrigido, refeito, preparado segundo um modelo biomédico da deficiência. Excluindo, dessa forma, inúmeros processos sociais e demandas significativas para a pessoa, reproduzindo açóes que incidem apenas na doença/deficiência e não em seus determinantes. Assim, situam as pessoas com deficiência como sujeitos individuais portadores de necessidades, deslocados das suas relações sociais e indiferenciados no que diz respeito à sua inserçáo de classe. Além disso, as instituiçóes de reabilitação apresentam pouca resolutividade em relaçáo às necessidades de saúde da população. 
No tocante à noção de necessidades de saúde, Campos e Bataeiro (2007) apontam duas vertentes conceituais que orientam atualmente a discussão sobre o tema: o conceito concreto operacional, que se configura como objeto dos processos de trabalho em saúde; e a perspectiva abstrata do conceito, considerada como aprimoramento da condiçáo humana. Neste trabalho, optou-se pelo conceito operacional, por contemplar a perspectiva dos sujeitos individuais e coletivos (STOTZ, 2004), na busca pela organizaçáo e produção dos serviços e ações em saúde que superem a racionalidade instrumental, ampliando, assim, o objeto de atenção à saúde. Nesse sentido, as necessidades são individualmente sentidas e são biológica e socialmente determinadas; sua atenção, satisfeita socialmente, é o sinal de seu reconhecimento. A noção de saúde aqui discutida é demarcada pela compreensão de que a saúde é influenciada por aspectos socioculturais e pelas condiçôes de vida resultantes da divisão da sociedade em classes e da concentração de renda e poder na mão de poucos.

Em geral, as necessidades de saúde são encaradas como necessidades sociais (CAMPOS; BATAEIRO, 2007), ou seja, são definidas como algo que se refere a todos ou à maioria que ocupa um dado território. Sendo as pessoas com deficiência "minoria" em um determinado território e se tratando de uma população historicamente excluída, suas necessidades passam a não ser levadas em conta. Assim, as necessidades de saúde das pessoas com deficiência são ocultadas e reprimidas, pois são percebidas como necessidades individuais e o planejamento das práticas de saúde para essa população é realizado com base em estudos epidemiológicos gerais (BRASIL, 2004), escassos e imprecisos.

Almeida, Tissi e Oliver (2000) aponta as enormes lacunas existentes no conhecimento tanto quantitativo quanto qualitativo sobre a situação das pessoas com deficiência. Além da carência de estudos que revelem o perfil epidemiológico dessa população, faltam também aqueles que se propóem investigar em profundidade as necessidades contextuais desse grupo, surgidas dos embates cotidianos em uma sociedade altamente excludente. Essas afirmaçōes nos levam a pensar que as informaçóes que norteiam o planejamento da atenção em saúde das pessoas com deficiência são abstratas, gerando, por conseguinte, açôes descontextualizadas em relação às realidades locais. Neste sentido, questiona-se: quem são as pessoas com deficiência e o que referem sobre suas necessidades de saúde em dado território da cidade de Salvador? Deste modo, este artigo objetiva traçar o perfil das pessoas com deficiências no tocante ao acesso a serviços e às necessidades de saúde, no intuito de discutir, sob uma dimensão coletiva, os cuidados oferecidos às pessoas com deficiência.

\section{Material e métodos}

Este estudo descritivo é oriundo de uma pesquisa mais ampla ${ }^{2}$ que buscou conhecer a situação de vida e saúde das pessoas com deficiência em Cosme de Farias, bairro pobre da cidade de Salvador. Consistiu na aplicação de questionários simplificados dirigidos a Agentes Comunitários de Saúde (ACS) de duas áreas de cobertura do Programa (PACS) no bairro, a fim de identificar pessoas com deficiência física e sensorial. Concluída esta etapa, iniciou-se a aplicação de questionários abertos dirigidos às pessoas identificadas, no intuito de aprofundar informaçóes sobre condiçôes de vida e infra-estrutura do bairro, situaçôes relacionadas à deficiência, atividades que as pessoas realizam e seu nível de participação social.

Para o presente trabalho, foram selecionadas, sobretudo variáveis que se referiam à caracterização das pessoas quanto ao perfil sócio-demográfico, tipo de deficiência, acesso aos serviços de saúde, necessidades e projetos de vida futuros. O banco de dados foi montado no Sistema Operacional Windows XP Professional, com o programa Microsoft Office Excel 2003, e foram realizadas análises dos dados com o objetivo de traçar o perfil geral dessa população a partir do recorte das questóes selecionadas para o estudo, assim como para obter a freqüência das variáveis (PINA, 2005), que posteriormente foram agrupadas em tabelas.

\section{Resultados e discussão}

\subsection{Caracterização sócio-demográfica}

Foram identificadas 235 pessoas com deficiência e/ou incapacidade motora e/ou sensorial numa área com população estimada em 20.000 habitantes. Os resultados apresentados na Tabela 1 revelam uma predominância de deficiência entre as mulheres $(63,8 \%)$. Tal dado afina-se com o de outros estudos de prevalência de deficiências e pode ser creditado, dentre outros fatores, ao fato de as mulheres apresentarem expectativa de vida mais longa, estando, portanto, mais propensas a limitaçôes funcionais características da idade avançada (SANTOS; SILVA; LESSA, 1989; ALMEIDA; TISSI; OLIVER, 2000; NERI et al., 2003; BRASIL, 2004; ROCHA; PAULA; KRETZER, 2004). Já os homens possuem 
Tabela 1. Caracterização sociodemográfica das pessoas com deficiência em Cosme de Farias, Salvador (BA), 2007.

\begin{tabular}{|c|c|c|}
\hline Variáveis do estudo faixa etária & $\mathbf{N}$ & $\%$ \\
\hline 0 a 04 anos & 1 & 0,43 \\
\hline 05 a 14 anos & 14 & 5,95 \\
\hline 15 a 24 anos & 14 & 5,95 \\
\hline 25 a 59 anos & 103 & 43,83 \\
\hline Acima de 60 anos & 103 & 43,83 \\
\hline Sexo & $\mathbf{N}$ & $\%$ \\
\hline Feminino & 150 & 63,8 \\
\hline Masculino & 85 & 36,2 \\
\hline Local de nascimento & $\mathbf{N}$ & $\%$ \\
\hline Salvador & 117 & 49,79 \\
\hline Interior da Bahia & 107 & 45,53 \\
\hline Outro estado & 11 & 4,68 \\
\hline Escolaridade & $\mathbf{N}$ & $\%$ \\
\hline Não alfabetizado & 43 & 18,3 \\
\hline Educação escolar incompleta & 118 & 50,21 \\
\hline Educação escolar completa & 24 & 10,21 \\
\hline $\begin{array}{l}\text { Outras (infantil, informal, } \\
\text { superior) }\end{array}$ & 50 & 21,28 \\
\hline Raça & $\mathbf{N}$ & $\%$ \\
\hline Negro & 127 & 54,04 \\
\hline Pardo & 82 & 34,89 \\
\hline Branco & 16 & 6,81 \\
\hline Outros & 10 & 4,26 \\
\hline Estado Civil & $\mathbf{N}$ & $\%$ \\
\hline Solteiro & 112 & 47,66 \\
\hline Casado & 58 & 24,68 \\
\hline Divorciado & 8 & 3,4 \\
\hline Viúvo & 56 & 23,83 \\
\hline Outros & 1 & 0,43 \\
\hline Renda familiar & $\mathbf{N}$ & $\%$ \\
\hline Menos de $1 / 2$ salário mínimo & 9 & 3,83 \\
\hline De $1 / 2$ a 3 salários mínimos & 208 & 88,51 \\
\hline Acima de 04 salários mínimos & 12 & 5,11 \\
\hline Não sabe informar & 6 & 2,55 \\
\hline
\end{tabular}

$4 \%$ a menos de chance de adquirirem deficiências (NERI et al., 2003).

A população adulta e idosa predomina, totalizando $87,6 \%$. Este dado confirma a tendência de aquisição de deficiências ao longo dos ciclos de vida e está relacionado ao aumento significativo de doenças crônico-degenerativas e casos de invalidez, nas últimas décadas, particularmente atingindo pessoas acima de 50 anos (ROCHA, 2006). Quando realizada a análise das variáveis sexo e idade, pode-se perceber que há um predomínio de mulheres adultas e idosas com deficiências (Figura 1).
Segundo os dados encontrados na caracterização da cor/raça, negros e pardos somaram $88,9 \%$ da população com deficiência, o que está diretamente relacionado à composição da população baiana, com predominância de afro-descendentes, sobretudo em Salvador. Nacionalmente, segundo o Censo de 2000, esse índice é menor, sendo negros e pardos com deficiência, $7,5 \%$ e 39,86\%, da população do país respectivamente (NERI et al., 2003). Esse é um aspecto de deve ser levado em conta na formulaçáo de políticas e planejamento de serviços e açóes para que estejam condizentes às necessidades de determinados grupos em territórios e regiôes específicas, haja vista que a população negra é, também, a mais pobre no país.

Com relação à proveniência da população, $49,7 \%$ refere nascimento em Salvador e um número semelhante de pessoas $(45,5 \%)$ do interior do estado. Esses números se justificam pela história de ocupação da cidade de Salvador, realizada, em grande medida, por pessoas que saíram do interior para a capital em busca de melhores condiçóes de saúde, educaçáo, moradia e emprego, e foram estabelecendo-se em áreas "desvalorizadas", de baixadas e encostas da cidade, submetendo-se a condições precárias de vida. A dificuldade de acesso a serviços básicos, como educação, também está presente no perfil dessa população, pois este mostra que mais da metade das pessoas possui educaçáo escolar incompleta $(50,2 \%)$ ou náo é alfabetizada (18,3\%). Investigaçōes sobre essa população apontam que "[...] quanto maior o número de anos de estudo, menor a proporção de pessoas com deficiência em relação à população não deficiente." (BRASIL, 2004), o que pode-se estabelecer uma relaçáo entre a falta de acesso à instrução e aquisição de deficiências. Não obstante, Neri et al. (2003) encontram em seu estudo um dado contrário, isto é, uma maior incidência no extrato populacional mais educado, que os autores atribuem ao fato de essas pessoas terem uma sobrevida maior. Esta discordância nos leva às seguintes conclusôes: a) a baixa escolaridade encontrada entre as pessoas com deficiência do bairro está diretamente relacionada ao nível de escolaridade da população em geral do bairro³ b) acredita-se que seja possível estabelecer relaçôes entre menor grau de instrução e aquisição de deficiência, visto que quem possui dificuldade de acesso à escola, possui conseqüentemente dificuldade para acessar outros serviços básicos (de saúde, sociais, de infra-estrutura, etc.), fato este relacionado diretamente à qualidade de vida.

Torna-se importante considerar o fato de o estudo ter sido realizado em um bairro pobre de uma cidade do Nordeste do Brasil, o que gerou 
Idade e sexo

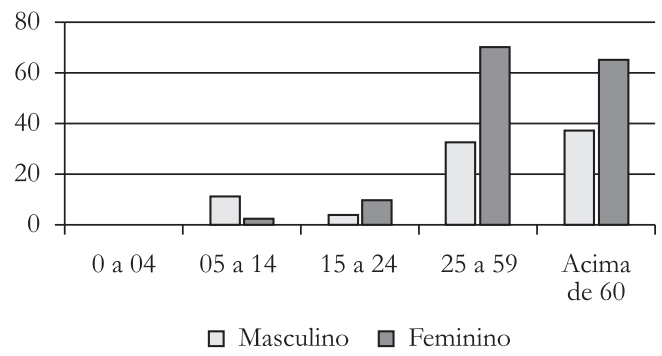

Figura 1. Relação entre faixa etária e sexo das pessoas com deficiência em Cosme de Farias, Salvador (BA), 2007.

dados que apontavam para uma estreita relação entre a pobreza e a deficiência. Coleridge, Simonnot e Steverlynck (2010) apontam que a aquisição de deficiências em países pobres estão diretamente relacionadas às condiçôes sanitárias, escassez de serviços básicos e problemas de saúde passíveis de prevenção, contudo ressaltam que há uma escassez que estudos que demonstrem essa relação.

Considerando que $88,5 \%$ possui renda familiar de $1 / 2$ a três salários mínimos, pode-se afirmar que essa população vive num contexto de baixo poder aquisitivo e situação de desigualdade social que reforça a perspectiva de bairro pobre. Pesquisas sobre população com deficiência (NERI et al., 2003) confirmam o pertencimento dessa populaçáo a classes mais empobrecidas e excluídas socialmente. Em "Retratos da Deficiência no Brasil" (NERI et al., 2003), dados analisados do Censo 2000 destacam a situação de desigualdade e desvantagem que estas pessoas enfrentam, especialmente desigualdades de raça (afro e indígena), gênero (mulheres), renda (miseráveis), educação (sem escola) e exclusão digital (sem computador). Percebe-se que a maior concentração de pessoas com deficiência está nas zonas urbanas e que os mesmos processos que determinam a estrutura da sociedade geram as desigualdades sociais e produzem perfis epidemiológicos de saúde e doença (BRASIL, 2004; NERI et al., 2003; COTTA et al., 2007).

Trata-se de uma população onde predominam grupos populacionais que historicamente conviveram com a exclusão social, como as mulheres, às quais cabe o "papel" dos afazeres domésticos e submissão ao homem; os idosos, geralmente taxados como inativos ou incapazes; os negros, que ainda vivem as desvantagens raciais herdadas do período da escravidão; as pessoas de baixa escolaridade, que não tiveram acesso à educação e às quais é oferecido o trabalho precarizado e de baixa remuneração e em ocupaçóes menos qualificadas. Além da população interiorana, oriunda da zona rural, que adaptam seus hábitos de vida e cultura para sobreviver na zona urbana.

\subsection{Atenção à saúde da pessoa com deficiência}

No que diz respeito ao tipo de deficiência (Tabela 2), há predominância da deficiência motora $(67,6 \%)$, seguida da deficiência visual $(12,7 \%)$ e múltipla (10,6\%). O percentual de deficiência/incapacidade motora é semelhante ao encontrado em outros estudos realizados nas cidades de São Paulo - SP, 63,3\% (ALMEIDA; TISSI; OLIVER, 2000) e Uberlândia - MG, 44,1\% (FERREIRA; BERTOLUCCI JUNIOR; CAMARGOS BORGES, 2006).

Quanto ao acesso aos cuidados de saúde, 80,4\% dos entrevistados referiram ter realizado tratamento. Destes (189), 49,2\% foram atendidos em instituiçóes hospitalares e 43,2\% em clínicas especializadas ou de reabilitação. A maior parte dessa população teve acesso à assistência gratuita (85,7\%). Observa-se, portanto, que se trata de uma população que utiliza o Sistema Único de Saúde ou é atendida por instituiçōes filantrópicas. Essas, ao longo da história, forneceram atenção caritativa e assistencial às pessoas com deficiência e atualmente é financiada em parte pelo SUS, onde disputam o mercado para garantir uma assistência de alto custo e tecnologia, ao contrário do que está previsto nas plataformas políticas.

Torna-se importante destacar que $60 \%$ das pessoas entrevistadas não realizavam mais tratamentos relacionados com a deficiência e 13\% nunca haviam sido submetidas a qualquer tipo de intervenção. Este dado é semelhante ao obtido em estudo em São Paulo, que mostra que $14,8 \%$ das pessoas não haviam tido acesso a nenhum tipo de assistência à saúde, no que se refere à deficiência (ALMEIDA; TISSI; OLIVER, 2000).

Foi também fundamental conhecer a dimensão da atenção em relação aos profissionais que ofereceram cuidado às pessoas com deficiência. Em geral, os médicos foram referidos como a especialidade profissional mais freqüente na busca por atendimento à deficiência. Para a pesquisa, foi necessário conhecer que outros profissionais, não médicos, como fisioterapeutas, terapeutas ocupacionais, psicólogos e fonoaudiólogos, também tiveram participação na prestação de serviços. Do total das pessoas entrevistadas, $6,38 \%$ referiram não ter recebido assistência de nenhum profissional, 34\% receberam assistência apenas do médico e $45,5 \%$ tiveram acesso a um profissional não médico, ou seja, menos da 
metade das pessoas com deficiência identificadas. Com base nas informaçóes referidas, pode-se obter um perfil da atençáo recebida, caracterizado por uma

Tabela 2. Caracterização por tipo de deficiência e modalidade da assistência em Cosme de Farias, Salvador (BA), 2007.

\begin{tabular}{|c|c|c|}
\hline $\begin{array}{l}\text { Variáveis do estudo } \\
\text { tipo de deficiência }\end{array}$ & $\mathbf{N}$ & $\%$ \\
\hline Motora & 159 & 67,66 \\
\hline Visual & 30 & 12,77 \\
\hline Auditiva & 21 & 8,94 \\
\hline Múltipla deficiência & 25 & 10,64 \\
\hline Realizou tratamento & $\mathbf{N}$ & $\%$ \\
\hline Sim & 189 & 80,43 \\
\hline Não & 46 & 19,57 \\
\hline Local onde realizou tratamento & $\mathbf{N}$ & $\%$ \\
\hline Apenas em hospital & 93 & 49,21 \\
\hline $\begin{array}{l}\text { Clínica especializada ou de } \\
\text { Reabilitação }\end{array}$ & 83 & 43,91 \\
\hline Outros & 13 & 6,88 \\
\hline Total & 189 & 100 \\
\hline Tipo de assistência recebida & $\mathbf{N}$ & $\%$ \\
\hline Gratuita & 162 & 85,71 \\
\hline Particular & 26 & 13,76 \\
\hline Outros & 1 & 0,53 \\
\hline Total & 189 & 100 \\
\hline Realiza tratamento atualmente & $\mathbf{N}$ & $\%$ \\
\hline Sim & 94 & 40 \\
\hline Não & 141 & 60 \\
\hline Total & 235 & 100 \\
\hline Local onde realiza tratamento & $\mathbf{N}$ & $\%$ \\
\hline Apenas em hospital & 50 & 53,19 \\
\hline $\begin{array}{l}\text { Clínica especializada ou de } \\
\text { reabilitação }\end{array}$ & 37 & 39,36 \\
\hline Outros & 7 & 7,45 \\
\hline Total & 94 & 100 \\
\hline $\begin{array}{c}\text { Tipo de assistência recebida } \\
\text { atualmente }\end{array}$ & $\mathbf{N}$ & $\%$ \\
\hline Gratuita & 64 & 68,09 \\
\hline Particular & 17 & 18,08 \\
\hline Outros & 13 & 13,83 \\
\hline Total & 94 & 100 \\
\hline $\begin{array}{l}\text { Especialidades profissionais } \\
\text { envolvidas no tratamento }\end{array}$ & $\mathbf{N}$ & $\%$ \\
\hline Somente médico & 80 & 34,04 \\
\hline Um profissional não médico & 107 & 45,53 \\
\hline Dois profissionais não médicos & 21 & 8,94 \\
\hline $\begin{array}{l}\text { Três ou mais profissionais não } \\
\text { médicos }\end{array}$ & 12 & 5,11 \\
\hline Nenhum profissional & 15 & 6,38 \\
\hline Total & 235 & 100 \\
\hline
\end{tabular}

atenção centrada na clínica médica especializada e não no trabalho de uma equipe de profissionais especialistas em reabilitação.

\subsection{Caracterização das necessidades e projetos de vida}

Uma parte considerável dos entrevistados $(86,8 \%)$ referiu necessidade de algum serviço de saúde voltado, ou sensível às pessoas com deficiência, no bairro (Tabela 3). A falta de assistência às pessoas com deficiência no próprio centro de saúde local foi motivo de queixa constante entre os entrevistados. Este fato pode estar atrelado a uma situação recorrente no trabalho de campo: durante a aplicação dos questionários, não era raro as pessoas apontarem para a falta de atendimento ou mesmo de procedimentos simples que poderiam ocorrer na própria unidade de saúde, particularmente pelo fato de serem deficientes. Todavia, dos entrevistados que referiram a necessidade de serviços de saúde, a maioria (47,5\%) considerou a falta de atenção específica relacionada à deficiência no local, por exemplo, aos comprometimentos das seqüelas e relacionada ao processo de reabilitação propriamente. A necessidade desses serviços especializados que se desenvolvam no bairro pode creditar-se ao alto percentual de pessoas que não recebe qualquer tipo de assistência deste tipo (60\%). Reflete também a defesa, por parte dessas pessoas, da descentralização do atendimento específico à pessoa com deficiência em Salvador. Esses serviços não devem estar concentrados apenas em locais centrais da cidade, altamente especializados, inacessíveis e distantes da realidade da população pobre, caracterizada, como mostra o perfil traçado, por muitas dimensôes de desigualdades e exclusáo. A relação entre o perfil dessa população e a necessidade de atendimento especializado remete à importância da equidade no acesso aos serviços de saúde. Nessa perspectiva, a condição de desvantagem para obtenção de cuidados à saúde deve ser minimizada para que as oportunidades de acesso sejam justas.

A melhoria na atenção à saúde para pessoas com deficiência no bairro está diretamente relacionada à melhoria de outros serviços básicos condizentes com uma vida digna. $45 \%$ das pessoas apontaram necessidades no que diz respeito aos serviços de saúde, segurança pública e infra-estrutura, além da dificuldade de acesso aos poucos serviços existentes, tais como transporte público, pavimentaçáo, adequação nas vias de acesso, entre outros. A geografia local, caracterizada por morros, baixadas, ladeiras e escadas no bairro, sem rampas ou corrimãos que favoreçam a locomoção, por exemplo, dificulta 
Tabela 3. Caracterização das necessidades de saúde e projetos de vida das pessoas com deficiência em Cosme de Farias, Salvador (BA), 2007.

\begin{tabular}{|c|c|c|}
\hline $\begin{array}{c}\text { Variáveis do estudo necessidade } \\
\text { de serviço no bairro }\end{array}$ & $\mathbf{N}$ & $\%$ \\
\hline Sim & 204 & 86,81 \\
\hline Não & 31 & 13,19 \\
\hline Total & 235 & 100 \\
\hline Necessidade de qual serviço? & $\mathbf{N}$ & $\%$ \\
\hline $\begin{array}{l}\text { Melhoria em relação a } \\
\text { serviços no bairro }\end{array}$ & 92 & 45,1 \\
\hline $\begin{array}{l}\text { Atendimento especializado } \\
\text { de saúde }\end{array}$ & 97 & 47,55 \\
\hline $\begin{array}{l}\text { Atividades de formação } \\
\text { (cursos, oficinas, palestras) }\end{array}$ & 15 & 7,35 \\
\hline Total & 204 & 100 \\
\hline Projetos de vida & $\mathbf{N}$ & $\%$ \\
\hline $\begin{array}{l}\text { Voltar a fazer o que fazia } \\
\text { antes da deficiência }\end{array}$ & 81 & 34,47 \\
\hline $\begin{array}{l}\text { Melhorias nas condições de } \\
\text { vida atuais }\end{array}$ & 49 & 20,85 \\
\hline $\begin{array}{l}\text { Trabalhar, estudar, cuidar e } \\
\text { ajudar a família, planos afetivos }\end{array}$ & 46 & 19,57 \\
\hline Sem projetos & 34 & 14,47 \\
\hline Outros & 25 & 10,64 \\
\hline Total & 235 & 100 \\
\hline
\end{tabular}

ainda mais o trânsito das pessoas pelo bairro e, consequentemente, sua saída dele. Tal dificuldade presente no meio urbano não impossibilita o acesso apenas das pessoas com deficiência, mas também todas as pessoas que em algum período da vida apresentam alguma condição de limitação, como gestantes e idosos (COVAS et al., 2003). A restrição de acesso a oportunidades, as dificuldades de circulação no bairro de residência e na cidade e as barreiras atitudinais configuram-se como potencializadores do isolamento e, como conseqüência, o acesso configura-se como uma das principais necessidades das pessoas com deficiência (OTHERO, 2010). A carência e a necessidade de melhoria dos serviços básicos e estruturais denunciam a iniquidade, desigualdade e náo acessibilidade enfrentada em um cotidiano de privaçóes.

No que concerne aos projetos de vida, o desejo mais salientado pelos entrevistados foi 'voltar a fazer o que fazia antes da deficiência' (34,4\%), seguido por 'melhorias nas condiçôes de vida'(20,8\%). Trabalhar, estudar, cuidar e ajudar a família foram desejos manifestados por $19,5 \%$ das pessoas entrevistadas. Othero (2010), em uma pesquisa realizada com pessoas com deficiência em São Paulo, também destaca que saúde, trabalho, educação e lazer foram aspectos abordados pelos entrevistados e afirma que as

[...] incapacidades advindas da deficiência fazem sobressair ainda mais a necessidade de autonomia, e também independência, sendo esta muito valorizada. (OTHERO, 2010, p. 97).

A análise desses dados, aliada à compreensão de que essas pessoas possuem importantes dificuldades em acessar serviços, apontam para o desejo de participação social, melhoria nas condiçôes de vida e resgate da vida laboral. Além disso, indicam necessidades que não são individuais, mas sim comuns aos demais moradores do bairro, pois estão relacionadas às condiçóes de vida no lugar.

Destacamos ainda que $14,4 \%$ das pessoas declararam não ter projetos de vida. Tal fato pode ser analisado levando em conta dois aspectos: o primeiro que se trata de uma populaçáo de baixa renda e marginalizada dos processos sociais, que convive diariamente com a ausência de oportunidades e condições para a própria sobrevivência; o segundo refere-se à própria exclusão decorrente da condição de deficiência que, na maioria das vezes, gera ausência de autonomia e de escolhas. Por conseguinte, muitas pessoas com deficiência não possuem planejamento para o futuro, pois a elas não são oferecidas outras possibilidades de viver.

\section{Considerações finais}

O presente estudo permitiu a identificação de uma populaçáo caracterizada por um complexo multifacetado de exclusão e privação coletiva, que inclui a pobreza; a desigualdade de gênero, raça e faixa etária; o náo acesso a serviços de educação, saúde e infra-estrutura e a precariedade das condiçóes de vida enfrentadas em áreas urbanas. Todos esses fatores acabam por perpetuar um típico circuito de retroalimentação constituído por desigualdade, pobreza e deficiência. O mundo contemporâneo - também excludente e desigual - configura-se, assim, como um grande produtor de deficiências e incapacidades, de modo que tais dimensóes interferem tanto na aquisição da deficiência, quanto na manutenção da desvantagem na inserção na vida social gerada pela interação desses fatores com a condição de deficiência.

A complexidade das questôes aqui levantadas sugere a necessidade de redirecionamento das práticas cotidianas em saúde no intuito de basear a organização do sistema de saúde na dialética da satisfação das necessidades. Para isso, considera-se que a Atenção Primária à Saúde seja relevante nesse processo. Vê-se 
ainda a necessidade de descentralização das açóes de reabilitação e de promoção da inclusão social, de forma que também possam acontecer próximas das moradias e da realidade social e política das pessoas com deficiência. Estas açóes podem ser orientadas pela estratégia da Reabilitação Baseada na Comunidade (RBC), compatível com a proposta da Atenção Primária à Saúde, conforme experiências de alguns municípios, como Sáo Paulo e Rio de Janeiro (OLIVER; ALMEIDA, 2007). As ações de $\mathrm{RBC}$ vinculadas aos serviços locais de saúde são propostas no documento Atençáo à pessoa com deficiência no Sistema Único de Saúde: planejamento e organização dos serviços, elaborado pelo Ministério da Saúde (BRASIL, 2009a) com o objetivo de subsidiar a capacitação de pessoal e instrumentalizar a implementação de programas no primeiro nível de atenção do Sistema Único de Saúde. Assim como estão contidas nas Diretrizes dos Núcleos de Apoio à Saúde da Família (BRASIL, 2009b) onde apontam como ação de apoio na área de reabilitação o desenvolvimento de propostas de Reabilitação Baseadas na Comunidade.

\section{Referências}

ALMEIDA, M. C.; TISSI, M. C.; OLIVER, F. C. Deficiência e Atenção Primária em Saúde: do conhecimento à invenção. Revista de Terapia Ocupacional da Universidade de São Paulo, São Paulo, v. 11, n. 1, p. 35-44, jan./abr. 2000.

BRASIL. Ministério da Justiça. Coordenadoria Nacional de Integração da Pessoa Portadora de Deficiência - CORDE. Relatório sobre a prevalência de deficiências, incapacidades e desvantagens. Niterói: CORDE, 2004.

BRASIL. Ministério da Saúde. Secretaria de Atenção à Saúde. Portaria n ${ }^{\circ}$ 1060, de 05 de junho de 2002. Aprova a Política Nacional de Saúde da Pessoa Portadora de Deficiência, e dá outras providências. Diário Oficial da República Federativa do Brasil, Brasília, DF, 6 jun. 2002. Seção 1.

BRASIL. Ministério da Saúde. Atenção à pessoa com deficiência no Sistema Único de Saúde: planejamento e organização dos serviços. Brasília: Ministério da Saúde, 2009a.

BRASIL. Ministério da Saúde. Secretaria de Atenção à Saúde. Departamento de Atenção Básica. Diretrizes do NASF - Núcleo de Apoio a Saúde da Família. Brasília: Ministério da Saúde, 2009b. (Série B. Textos Básicos de Saúde; Cadernos de Atenção Básica, n. 27).

CAMPOS, C. M.; BATAEIRO, M. O. Necessidades de saúde: uma análise da produção científica brasileira de 1990 a 2004. Interface: Comunicacao, Saude, Educacao, Botucatu, v. 11, n. 23, p. 605-18, set./dez. 2007.

COLERIDGE, P.; SIMONNOT, C.; STEVERLYNCK, D. Study of Disability in EC Development cooperation. Brussels: European Commission, 2010. 127 p. Final Report (draft).
COTTA, R. M. M. et al. Pobreza, Injustiça e Desigualdade Social: repensando a formação de profissionais de Saúde. Revista Brasileira de Educação Médica, Rio de Janeiro, v. 31, n. 3, p. 278-286, set./dez. 2007. http://dx.doi. org/10.1590/S0100-55022007000300010

COVAS, C. A.; AKASHI, L. T.; GARCIA, M. A. Avaliação da qualidade dos espaços urbanos sob o ponto de vista das pessoas com deficiência. Cadernos de Terapia Ocupacional da UFSCar, São Carlos, v. 11, n. 1, p. 5-20, 2003.

DINIZ, D.; BARBOSA, L.; SANTOS, W. Deficiência, Direitos Humanos e Justiça. Sur: Revista Internacional de Direitos Humanos, São Paulo, v. 6, n. 11, p. 65-77, dez. 2009. http://dx.doi.org/10.1590/ S1806-64452009000200004

FERREIRA, E. W.; BERTOLUCCI JUNIOR, L.; CAMARGOS BORGES, M. M. Perfil Demográfico e do Emprego das Pessoas Portadoras de Deficiência: população dependente de políticas públicas na ilha de prosperidade de Uberlândia-MG. In: ENCONTRO NACIONAL DE ESTUDOS POPULACIONAIS, 15., 2006, Caxambu. Anais... ABEP: Caxambu, 2006. p. 1-21. Mesa Redonda - Pessoas Portadoras de Deficiência.

GOFFMAN, E. Estigma: notas sobre a manipulação da identidade deteriorada. Rio de Janeiro: Zahar, 1963. INSTITUTO BRASILEIRO DE GEOGRAFIA E ESTATÍSTICA - IBGE. Censo demográfico 2000. Rio de Janeiro: IBGE, 2000.

MAIOR, I. M. M. L. Políticas públicas sociais para as pessoas portadoras de deficiência no Brasil. Cadernos de Pesquisa / Centro Brasileiro de Analise e Planejamento, São Paulo, n. 7, p. 31-47, maio 1997. Disponível em: <http:// www.cebrap.org.br/v2/files/upload/biblioteca_virtual/ politicas_publicas_sociais.pdf >. Acesso em: 03 out. 2007.

NERI, M. C. et al. Retratos da deficiência no Brasil. Rio de Janeiro: FGV; IBRE; CPS, 2003.

OLIVER, F. C.; ALMEIDA, M. C. Reabilitação Baseada na Comunidade. In: CAVALCANTI, A.; GALVÃO, C. (Orgs.). Terapia Ocupacional: fundamentação \& prática. Rio de Janeiro: Guanabara Koogan, 2007. p. 125-132.

ORGANIZAÇÃO MUNDIAL DA SAÚDE - OMS. Centro colaborador da Organização Mundial da Saúde para a Família de Classificaçôes Internacionais. CIF: Classificação Internacional de Funcionalidade, Incapacidade e Saúde. São Paulo: EdUSP, 2003.

OTHERO, M. B. Atenção à saúde da pessoa com deficiência: necessidades sob a perspectiva dos sujeitos. 2010. $331 \mathrm{f}$. Dissertação (Mestrado em Ciências)-Faculdade de Medicina, Universidade de São Paulo, São Paulo, 2010.

PINA, A. P. B. Investigação e Estatística com o EpiInfo. Lisboa: Gabinete de Investigação e Estatística, Delegação Regional do Algarve do Instituto da Dorga e Toxicodependência, 2005. Disponível em: <http://www. saudepublica.web.pt/03-investigacao/031-epiinfoinvestiga/ plano.htm>. Acesso em: 14 nov. 2011.

ROCHA, E. F. Reabilitação de pessoas com deficiência: a intervenção em discussão. São Paulo: ROCA, 2006. 
ROCHA, E. F.; PAULA, A. R.; KRETZER, M. R. O estudo de prevalência de deficiências e incapacidades como instrumento de planejamento das atividades de atenção à saúde e reabilitação no Programa de Saúde da Família. Revista de Terapia Ocupacional da Universidade de São Paulo, São Paulo, v. 15, n. 1, p. 1-10, jan./abr. 2004. SANTOS, J. R.; SILVA, A. C.; LESSA, I. Prevalência de incapacidades em dois diferentes grupos sociais em Salvador, Brasil. Boletim da Oficina Sanitária Pan-Americana, Washington, v. 106, n. 4, p. 304-313, abr. 1989. Disponível em: <http://hist.library.paho.org/ Spanish/BOL/v106n4p304.pdf>. Acesso em: 08 out. 2007
STOTZ, E. N. Os desafios do SUS e a Educação Popular: uma análise baseada na Dialética da Satisfação das Necessidades de Saúde. In: BRASIL. Ministério da Saúde. Secretaria de Gestáo do Trabalho e da Educação na Saúde. Departamento de Gestão da Educação na Saúde. Ver-SUS Brasil: caderno de textos. Brasília: Ministério da Saúde, 2004.

TEIXEIRA, C. F.; PAIM, J. S.; VILASBOAS, A. L. SUS: Modelos Assistenciais e Vigilância da Saúde. Informe Epidemiológico do SUS, Brasilia, v. 7, n. 2, p. 7-28, abr./ jun. 1998 .

\section{Contribuição dos Autores}

As autoras participaram de todas as etapas de construção deste artigo e trabalharam de forma colaborativa desde a concepção do texto manuscrito até a redação e revisão do mesmo.

\section{Notas}

${ }^{1}$ Projeto "Mapeando Territórios: a situação de vida e saúde das pessoas com deficiência", desenvolvido entre os anos de 2005 e 2007 pelo Curso de Terapia Ocupacional da Escola Bahiana de Medicina e Saúde Pública e sob coordenação das professoras Adriana Miranda Pimentel e Maria Tereza Baraúna da Costa.

${ }^{2}$ Projeto "Mapeando Territórios: a situação de vida e saúde das pessoas com deficiência", desenvolvido entre os anos de 2005 e 2007 pelo Curso de Terapia Ocupacional da Escola Bahiana de Medicina e Saúde Pública e sob coordenação das professoras Adriana Miranda Pimentel e Maria Tereza Baraúna da Costa.

${ }^{3}$ Cerca de $1 / 4$ da população do bairro não tem instrução formal ou tem menos de um ano de estudo, e mais da metade dos chefes de família cursaram apenas o ensino fundamental (IBGE, 2000). 